\title{
È ancora attuale il tema dell'appropriatezza prescrittiva nella gestione del paziente con infezione da HIV?
}

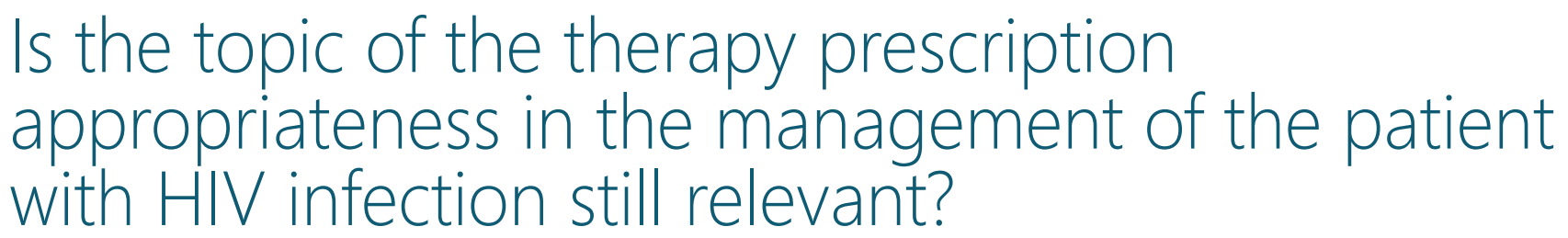

\section{Davide Croce, Frida Milella}

Centro di ricerca in economia e management in sanità e nel sociale, Università Carlo Cattaneo - LIUC

\section{Riassunto}

Anche se l'accessibilità dei pazienti alla terapia antiretrovirale (HAART) non è ancora sovrapponibile in tutto il mondo, l'infezione da HIV è rapidamente diventata una malattia cronica che garantisce una elevata qualità di vita. Ciò nonostante la ricerca sia continuamente tesa all'obiettivo di proporre nuovi farmaci a partire dalle necessità legate alle caratteristiche del paziente. In Italia paziente "tipico" è di genere maschile e la sua infezione è databile nell'età adulta piuttosto che in quella giovanile; sempre più frequentemente presenta patologie croniche.

I problemi che sorgono in una popolazione così composta riguardano la trattamento nel tempo, il monitoraggio del paziente e la definizione dell'organizzazione per assistere in modo efficace una coorte tendenzialmente in una fascia di età più avanzata.

L'organizzazione del lavoro vede due modelli principali: assistenza attraverso un team multidisciplinare oppure attraverso un progressivo allargamento delle competenze dei medici che hanno in carico i pazienti.

Nella discussione fra questi due modelli è necessario tenere in considerazione che nuovi tipi di trattamento saranno probabilmente rapidamente disponibili con l'intento di migliorare l'aderenza e la qualità di vita del paziente. La proposta delle nuove terapie dovrà però tenere conto ancora una volta della storia clinica e farmacologica del paziente.

$E^{\prime}$ quindi verosimile che, come indicato anche dal piano nazionale, la presa in carico da parte di uno specialista e la personalizzazione del trattamento continueranno ad essere un elemento importante del modello di assistenza e del paziente con infezione da HIV.

\section{Introduzione}

L'HIV rappresenta uno dei casi di brillante successo della medicina. La cura farmacologica è passata in pochi anni da supporto e accompagnamento all'evento infausto, a malattia cronica da gestire

\section{Abstract}

Although the accessibility of patients to antiretroviral therapy (HAART) is not yet overlapping worldwide, HIV infection has rapidly become a chronic disease that guarantees a high quality of life. The HIV therapy research is constantly aimed at proposing new drugs based on the needs related to the patient's characteristics. In Italy "typical" patient is male gender and its infection is datable in adulthood rather than in juvenile; more and more frequently it presents chronic pathologies.

The problems that arise in such a composite population are related to time management, patient monitoring and organization definition to effectively assist a cohort tending to a more advanced age group.

The organization of work presents two main models: assistance through a multidisciplinary team or through a progressive enlargement of the skills of the doctors who are in charge of the patient. To choose between these two models it is necessary to take into consideration that new types of treatment will probably be rapidly available with the aim of improving the patient's adherence and quality of life. The proposal of new therapies, however, will have to take account of the patient's clinical and pharmacological history once again.

It is therefore likely that, as also indicated by the chronic diseases national plan, the taking charge by a specialist and the personalization of the treatment will continue to be an important element of the model of care and of the patient with HIV infection.

\section{Corrisponding Author:}

\section{Davide Croce}

Centro di ricerca in economia e management in sanità e nel sociale, Università Carlo Cattaneo - LIUC

dcroce@liuc.it

Keywords:

Health-Related Quality of Life (HRQoL); Fourth Ninety (4th 90); comorbidities; self-perceived quality of life (self-perceived QoL); survey (questionnaire).

\section{Potenzial conflict}

of interest: see the end of the article

JHA 2018; S1: 5-8

DOI: 10.19198/JHA31463 con completa compatibilità ad una vita normale del paziente. Il successo non è ancora tale in tutto il mondo, a causa dell'accessibilità alla terapia (1), della quantità e qualità dell'assistenza sanitaria in alcune aree del mondo (Figura 1 -2). 


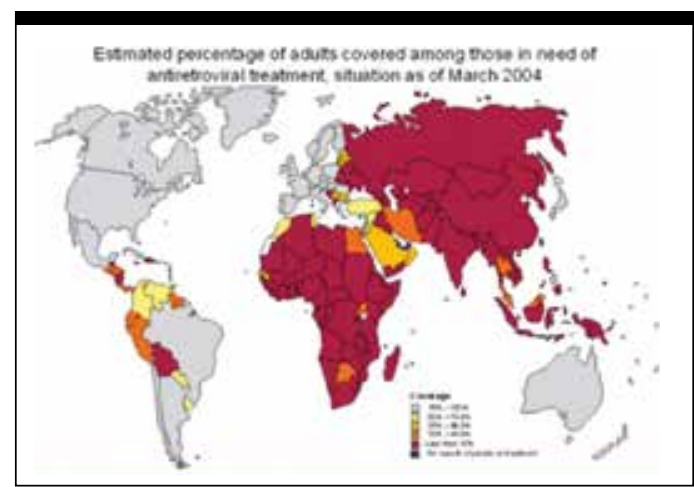

Figura 1. 2004, stima della percentuale di copertura sul trattamento HIV

Tuttavia questo grande successo non ha rallentato la ricerca sulle cure, con l'obiettivo di migliorare la qualità della vita dei pazienti, inserendosi con questo filone anche nei cambiamenti in atto nella popolazione dei Paesi ad economia sviluppata.

Questi due temi, ovvero lo sviluppo della terapia farmacologica e le caratteristiche della popolazione servita, saranno le logiche di analisi della risposta al quesito se è ancora attuale l'appropriatezza prescrittiva nella gestione del paziente affetto da HIV, domanda non retorica alla luce di quanto oggi accade nella cura di un altro virus, quello da epatite $C$, dove lo sviluppo della terapia farmacologica ha di fatto standardizzato la cura con le terapie pangenotipiche.

\section{Le caratteristiche della popolazione italiana}

L'Italia è un Paese sempre più anziano, come si evince dalla Figura 3.

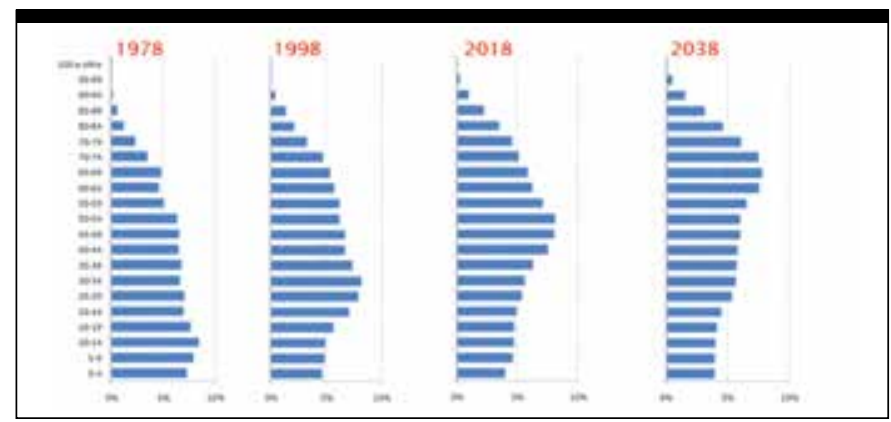

Figura 3. Composizione popolazione italiana (elaborazione su dati ISTAT)

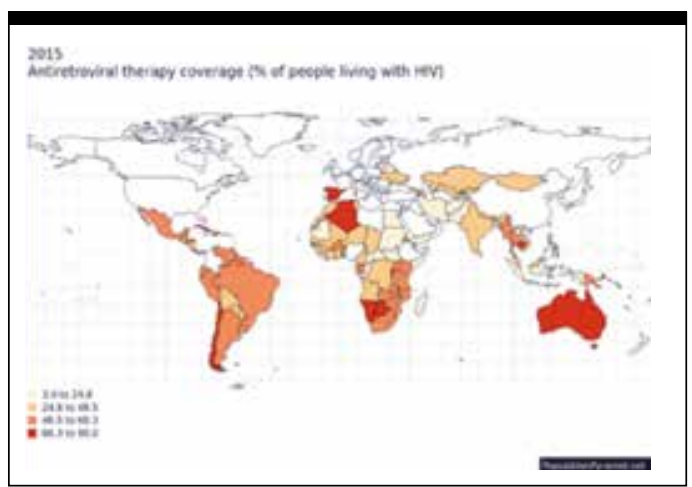

Figura 2. 2015, stima UNAIDS della percentuale di copertura sul trattamento HIV.

I dati elaborati dalla Regione Veneto (2) ci forniscono due elementi più o meno comuni alla popolazione italiana affetta da virus HIV (PLWHIV): il rapporto maschi/femmine è passato da 2:1 a circa 3:1, l'età media prevalente d'infezione si è trasferita nella sua componente preponderante da 25-34 anni a 25-44 anni, e l'età della prevalenza si è spostata da 35-44 a 45-64 anni (Figura 4 - 5).

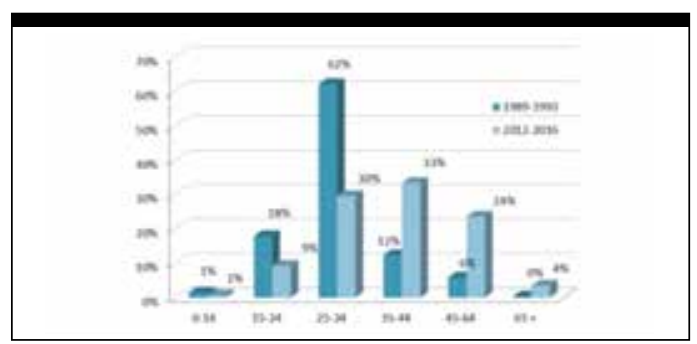

Figura 4. Età alla diagnosi delle nuove infezioni, Regione Veneto 2016

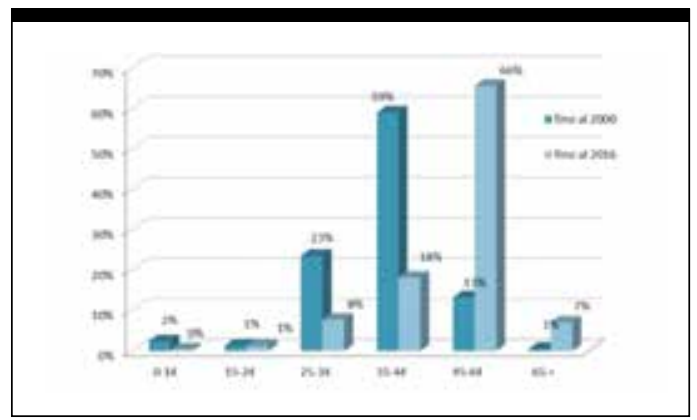

Figura 5. Prevalenza in classi di età nella coorte veneta al confronto 2000 e 2016 
Sulla base delle caratteristiche della PLWHIV della Regione Veneto possiamo dire che il paziente "tipico" si colloca nell'età in cui l'incidenza delle patologie croniche vede il suo avvio, è di genere maschile e che la sua infezione è databile nell'età adulta piuttosto che in quella giovanile, essendosi allungata nei fatti la vita dei PLWHIV. La popolazione PLWHIV si espone sempre più frequentemente quindi alle patologie croniche, dal diabete ai problemi cardiovascolari, in una positiva "normalità" di sovrapposizione con la popolazione generale.

I problemi che sorgono in una popolazione così composta riguardano la terapia e il controllo, come pure l'organizzazione per assistere in modo efficace una coorte anziana.

L'organizzazione del lavoro da mettere in campo per l'assistenza di una popolazione affetta da una patologia principale importante e altre patologie croniche concomitanti vede due modelli principali (3):

- assistenza attraverso un team multidisciplinare;

- assistenza attraverso un progressivo allargamento delle competenze dei medici che hanno in carico la coorte di PLWHIV.

Il primo caso vede una forte componente di allineamento dei processi di presa in carico per la cura, con particolare riferimento al tempo, e risultati tendenzialmente positivi in outcome in breve tempo se il team è efficiente nel lavoro di assistenza. L'approccio per implementation science (4) è particolarmente importante.

Il secondo caso vede un grosso carico di responsabilità verso il singolo, che deve nel tempo allargare le proprie competenze. II vantaggio del modello singolo è la certezza della presa in carico, mentre lo svantaggio è la disomogeneità dell'assistenza legata alla conoscenza del medico. Infine una lettura delle comorbilità.

Secondo l'osservatorio epidemiologico della Regione Lombardia (5) le cronicità associate all'HIV in termini di consumo di DDD nel 2017 sono state:

a) apparato gastrointestinale e metabolismo

b) cardiovascolare

c) sangue e organi emopoietici

d) sistema nervoso

e) sistema muscolo-scheletrico

f) sistema genito-urinario ed ormoni sessuali

g) sistema respiratorio h) organi di senso

i) neoplasie

Con una netta prevalenza delle prime tre indicazioni.

\section{La tecnologia sanitaria per la cura all'HIV}

L'elevata efficacia, la sicurezza, la tollerabilità e la comodità della terapia ARV contemporanea, difficilmente ci porta ad identificare dove e come saranno apportati miglioramenti. Tuttavia, i vasti progressi nei trattamenti in tutte le aree terapeutiche ci indicano che l'assunto è nelle agende delle case farmaceutiche.

Il primo passaggio di sfida all'attuale paradigma terapeutico, basato su due/tre farmaci antiretrovirali attivi per via orale assunti giornalmente per mantenere la soppressione virale, è dato dalle due opzioni di terapia farmacologica che possono semplificare il trattamento e ridurre i costi.

Il secondo passaggio sono i farmaci a lunga durata somministrati ogni settimana o ogni mese, che possono facilitare la cura per alcune categorie di pazienti, migliorandone l'aderenza al trattamento. Alcuni farmaci antiretrovirali ad azione prolungata in fase di sviluppo forniranno ulteriori opzioni di terapia orale, ma la maggior parte sarà probabilmente erogata attraverso sistemi di somministrazione alternativa di farmaci. Ciò include la dapivirina all'interno di un anello vaginale, e tenofovir alafenamide come impianto subdermico. Questi metodi di somministrazione del farmaco possono sembrare particolari per I'HIV, ma sono metodi comuni per somministrare farmaci in altre aree terapeutiche e possono rappresentare un metodo nuovo ed efficace per garantire l'aderenza al farmaco e un trattamento efficace.

Nuovi agenti, con nuovi bersagli terapeutici, possono sfidare lo status quo terapeutico. Assemblaggio del capside, attaccamento della glicoproteina 120 ed espressione dell'mRNA Revdipendente sono tra i nuovi bersagli della terapia farmacologica nell'ambito degli studi in corso. Gli agenti in queste classi, insieme alla recente approvazione di un nuovo anticorpo monoclonale anti-CD4, possono fornire opzioni terapeutiche future, in particolare per quelli con lunga esperienza di trattamento farmacologico. 
L'ultimo componente del paradigma terapeutico riguarda la costante necessità di nuovi farmaci antiretrovirali e potrebbe essere il più difficile da cambiare. La neutralizzazione di anticorpi, vaccini terapeutici, agenti di inversione della latenza o la tecnologia CRISPR (clustered regularly interspaced short palindromic repeats) potrebbero diventare i metodi più comuni per inibire, sopprimere o eliminare I'HIV. Le prove di questi approcci e di altri continuano ad emergere e forniscono ottimismo sul fatto che il futuro possa contenere una potenziale cura.

In questa veloce disanima si osserva come difficilmente possa emergere una cura standard per tutti i pazienti, che sono differenti per età, genere, comorbilità, approccio alla cura (e.g. aderenza e persistenza).

\section{Conclusioni}

Se le caratteristiche del paziente (PLWHIV) si stanno modificando verso uno stato di salute abbinato a patologie croniche e contemporaneamente la tecnologia sanitaria si orienta verso una personalizzazione della terapia farmacologica, appare evidente comela semplificazione terapeutica sia una chimera data dalla attuale disponibilità di terapie che con alta efficacia permettono approcci semplificati rispetto al passato. Soggetti più vecchi con terapie specifiche per target inducono ad una maggiore personalizzazione.

Tuttavia il ragionamento continua ad essere fatto sul paziente naive: il paziente experience ancora viene preso poco in considerazione negli studi clinici ed è lasciato all'esperienza del singolo medico trattante, con le conseguenze sulle coorti che si possono immaginare. Forse in questa categoria di popolazione si potrà immaginare l'uso di trattamenti farmacologici standard legati alle nuove molecole in commercio da pochi anni.

In generale, si sottolinea come la personalizzazione sia un elemento importante del modello di presa in carico della cronicità, ribadito anche dal piano nazionale (6). E la presa in carico, con tutte le personalizzazioni che le competono, è uno dei punti di forza della cura offerta ai PLWHIV italiani, che quindi risultano più aderenti e persistenti degli analoghi statunitensi.

\section{REFERENCES}

1. Gerberding J Steps on the Critical Path: Arresting HIV/AIDS in Developing Countries. PLoS Med 2004; 1(1): e10

2. Regione Veneto, Registro HIV, Dati al 31 Dicembre 2016. Disponibile al sito http://repository.regione.veneto.it/ public/3aa14507ad0dac2f5279ce0f5cd71777.php. Ultimo accesso 15 novembre 2018

3. D Croce, D Mueller, G Rizzardini, U Restelli, Organising HIV ageing-patient care in South Africa: An implementation science approach. Southern African Journal of Public Health 2018; 2:59-62

4. Bauer MS, Damschroder L, Hagedorn $\mathrm{H}$, et al An introduction to implementation science for the non-specialist. BMC Psychol. 2015; 16: 3-32.

5. Dati di consumo dei pazienti affetti da HIV per Regione Lombardia. Disponibili al sito: https://www.dati.lombardia.it/Sanit-/Dataset-consumifarmaceutica-pazienti-cronici/4nv7-wwhh. Ultimo accesso 15 novembre 2018

6. Piano nazionale della cronicità. Ministero della Salute. Disponibile al sito http://www.salute.gov.it/imgs/C_17_pubblicazioni_2584_allegato. pdf. Ultimo accesso 15 novembre 2018 